\title{
Corrigendum: Genome-wide association study of colorectal cancer identifies six new susceptibility loci
}

Fredrick R. Schumacher, Stephanie L. Schmit, Shuo Jiao, Christopher K. Edlund, Hansong Wang, Ben Zhang, Li Hsu, Shu-Chen Huang, Christopher P. Fischer, John F. Harju, Gregory E. Idos, Flavio Lejbkowicz, Frank J. Manion, Kevin McDonnell, Caroline E. McNeil, Marilena Melas, Hedy S. Rennert, Wei Shi, Duncan C. Thomas, David J. Van Den Berg, Carolyn M. Hutter, Aaron K. Aragaki, Katja Butterbach, Bette J. Caan, Christopher S. Carlson, Stephen J. Chanock, Keith R. Curtis, Charles S. Fuchs, Manish Gala, Edward L. Giovannucci, Stephanie M. Gogarten, Richard B. Hayes, Brian Henderson, David J. Hunter, Rebecca D. Jackson, Laurence N. Kolonel, Charles Kooperberg, Sébastien Küry, Andrea LaCroix, Cathy C. Laurie, Cecelia A. Laurie, Mathieu Lemire, David Levine, Jing Ma, Karen W. Makar, Conghui Qu, Darin Taverna, Cornelia M. Ulrich, Kana Wu, Suminori Kono, Dee W. West, Sonja I. Berndt, Stephane Bezieau, Hermann Brenner, Peter T. Campbell, Andrew T. Chan, Jenny Chang-Claude, Gerhard A. Coetzee, David V. Conti, David Duggan, Jane C. Figueiredo, Barbara K. Fortini, Steven J. Gallinger, W. James Gauderman, Graham Giles, Roger Green, Robert Haile, Tabitha A. Harrison, Michael Hoffmeister, John L. Hopper, Thomas J. Hudson, Eric Jacobs, Motoki Iwasaki, Sun Ha Jee, Mark Jenkins, Wei-Hua Jia, Amit Joshi, Li Li, Noralene M. Lindor, Keitaro Matsuo, Victor Moreno, Bhramar Mukherjee, Polly A. Newcomb, John D. Potter, Leon Raskin, Gad Rennert, Stephanie Rosse, Gianluca Severi, Robert E. Schoen, Daniela Seminara, Xiao-Ou Shu, Martha L. Slattery, Shoichiro Tsugane, Emily White, Yong-Bing Xiang, Brent W. Zanke, Wei Zheng, Loic Le Marchand, Graham Casey, Stephen B. Gruber \& Ulrike Peters

Nature Communications 6:7138 doi: 10.1038/ncomms8138 (2015); Published 7 Jul 2015; Updated 26 Oct 2015

The original version of this Article contained typographical errors in the spelling of the authors Sébastien Küry, Edward L. Giovannucci and Mathieu Lemire, which were incorrectly given as Sebastian Kury, Edward L. Giocannucci and Mathiew Lemire. This has now been corrected in both the PDF and HTML versions of the Article. 